\title{
ВАЛЮТНЫЙ РИСК И ЛОКАЛЬНАЯ МОДЕЛЬ ЦЕНООБРАЗОВАНИЯ АКТИВОВ НА ПРИМЕРЕ РОССИЙСКОГО РЫНКА АКЦИЙ
}

Кучин Илья Игоревич ${ }^{1}$

Данное исследование посвящено проблеме выявления влияния факторов риска на ценообразование активов на российском рынке. Джон Кэмбелл в 2013 году разработал для американского рынка межвременную модель ценообразовани активов, учитывающую ряд факторов риска. Развивающиеся рынки капитала, в том числе и российского, имеют свои особенности, которые усложняют использование глобальных факторов риска локальной модели ценообразования. Присутствие дополнительных рисков, связанных с неэффективностью рынка, ограниченными возможностями диверсификации, структурными и институциональными особенностями развивающихся стран, требует выбора других ключевых факторов, влияющих на ценообразование активов, а также использования локальных моделей для оценки требуемой доходности на собственный капитал. В частности, в качестве факторов риска на российском рынке были выбраны взаимосвязанные и ключевые для российского рынка параметры валютного курса и цены на нефть. Тестирование модели проводилось на данных за 2005-2013 годы по 102 нефинансовым российским компаниям. Новизна в данной работе заключалась в следующем: во-первых, разработана новая модель оценки фактора влияния валютного риска на затраты на собственный капитал; во-вторых, произведен учет влияния валютного курса на риск колебаний денежных потоков, ставки дисконтирования и идиосинкратическую волатильность в межвременной модели ценообразования активов; в-третьих, проведено тестирование межвременной модели ценообразования активов на российском рынке. В результате подтвердилась сильная спецификация модели, что говорит о возможности её использования и необходимости учета макрофакторов при формировании портфеля для инвестирования в долгосрочной перспективе.

Ключевые слова: межвременная модель ценообразования, валютный риск, локальная модель, MGARCH

JEL: G12

\section{Введение}

В 80-е годы XX века исследователи обнаружили, что модель ценообразования активов САРМ Шарпа и Литнера не может объяснить поведение доходности акций. А также при помощи эмпирического тестирования было установлено, что статистически значимым образом акции с малой капитализацией превосходят акции с большей капитализацией [Banz, 1981]. Таким образом, последующие исследования были посвящены проверке корректности использования модели САРМ. В частности, САРМ является статической моделью, в которой ожидаемая доходность акций считается постоянной. Однако, если ожидаемая доходность является изменяющейся во времени, то доходность активов определяется не только в зависимости от доходности фондового рынка, как в САРМ, но и от переменных, которые прогнозируют доходность рынка [Merthon, 1973; Campbel, 2011].

В работе Мертона [Merthon, 1973] разрабатывается межвременная модель ценообразования активов. Фама и Френч - одни из первых исследователей, которые стали использовать и модифицировать методологию межвременной модели ценообразования для решения проблемы с прогнозированием доходности акций. Авторы ввели в модель дополнительные факторы, влияющие на доходность акций рынка США (валютный курс, кредитный дефолтный своп, волатильность рынка и другие факторы) [Fama, French, 1989]. Также было установлено, что колебания макропеременных могут оказывать как отрицательное, так и положительное влияние на доходность каждой компании, особенно на развивающихся рынках, ввиду их нестабильной экономической ситуации в последние годы. Поэтому выявление основных локальных факторов риска является ключевой задачей в прогнозировании ожидаемой доходности компаний развивающихся рынков, в том числе и России.

${ }^{1}$ Аспирант НИУ ВШЭ, г. Москва, ул. Шаболовка, д. 26. E-mail: ikuchin@hse.ru 
Кэмпбелл в 1987-м и Фама, Френч в 1989 году считали, что краткосрочные процентные ставки и дивидендная доходность прогнозируют доходность рыночного портфеля [Campbell, 1987; Fama, French, 1989]. Интересно, что Гуо в 2003 году обнаружил, что предсказательная сила модели существенно улучшается, если дисперсия рыночного портфеля и стохастическая безрисковая ставка включаются в уравнение прогнозирования [Guo, 2003]. Причем последняя переменная предоставляет дополнительную информацию о будущей прибыли [Guo, 2003].

Боссертс и Хиллион в 1999 году обнаружили, что предсказательная сила прогноза доходности акций незначительна [Bossaerts, Hillion, 1999]. Аналогично Шверт в 2002 году считал, что многие торговые стратегии, которые были найдены для создания сверхдоходности, были убыточны в прошлом десятилетии [Shchwert, 2002]. В целом Малкил в 2003 году утверждал, что нет никаких достоверных свидетельств уверенной прогнозируемости доходности акций и что американский фондовый рынок эффективен в том смысле, что сверхдоходности быстро исчезают после их обнаружения [Malkiel, 2003].

Кроме того, авторы Леттау и Людвигсон в 2001 году, показывают, что отношение потребления к благосостоянию, особенно в сочетании с волатильностью фондового рынка, являются статистически и экономически значимыми величинами для прогнозирования ожидаемой доходности рыночного портфеля. Резонно полагать, как утверждает Кэмпбелл, что доходность акций есть прогнозируемые колебания [Lettau, Ludvigson, 2001; Campbell, 2000].

Поведенческие финансы быстро развиваются с 1990 года, и Шиллер в 2003 году, в частности, подчеркнул их важную роль в восстановлении современных финансов. Основная критика состояла в том, что длинный список когнитивных искажений дает исследователям так много степеней свободы, что ими все можно объяснить. Но финансовые экономисты больше интересуются прогнозом, лежащим вне образца, а именно: «Как объяснить, что произошло?» Кроме того, в это трудно поверить, что инвесторы могут выжить в арбитраже, управляемом финансовым рынком.

Как уже упоминалось, прогнозируемость доходности акций имеет важное значение в ценообразовании активов. Фама в 1991 году предположил, что мы должны использовать ожидаемую доходность, как величину, изменяющуюся во времени [Fama, 1991]. В соответствии с этой теорией некоторые современные авторы считают, что предсказанные значения индекса фондового рынка и его волатильности действительно помогают объяснить ожидаемую доходность акций [Campbell, Vuolteenaho, 2002]. Примерно по такому же пути следовали Ду и другие [Du, Ng, Zhao, 2013], которые объединили идеи Фрэнсиса и соавторов [Francis et al., 2008], предложив наряду с доходностью рыночного портфеля и изменениями валютного курса ввести два типа дополнительных переменных. Один тип переменных соответствовал специфическим для компании факторам, а второй - внешним. Причем оба влияли непосредственно на подверженность валютному риску. Чаиб и Мазотта [Chaieb and Mazzotta, 2013] протестировали на панельных данных модель, в которой изменение подверженности во времени также связано с экзогенными и эндогенными параметрами, такими как спреды процентных ставок, рычаг и ликвидность. Результаты различных тестов условных динамических моделей Фрэнсиса, Чаиб и Мазотта, Ду и др. [Francis et al., 2008; Chaieb and Mazzotta, 2013; $\mathrm{Du}, \mathrm{Ng}$, Zhao, 2013] показали значительное влияние валютных рисков в некоторых отраслях американской экономики.

Шверт в 1989 году, как и многие другие, находит кластеризацию волатильности на фондовом рынке. Автор выявил следующую закономерность: при увеличении волатильности кластеризация остается на высоком уровне в течение длительного периода времени. Суть данного исследования состоит в том, что в дополнение к премии за рыночный риск инвесторы также требуют премию за ликвидность акций, потому что они не могут использовать акции для хеджирования своих рисков в связи с ограниченным количеством участников фондового рынка. Таким образом, волатильность акций и коэффициент отношения потребления к благосостоянию прогнозируют доходность фондового рынка [Schwert, 1989].

Кэмпбэл и Волтенахо [Campbell, Vuolteenaho, 2004] разработали модель ценообразования, в 
которой ковариация доходностей с факторами риска определялась через динамику внешних финансовых переменных. В таком же направлении пройдет и наше исследование. В текущей работе объектом будет служить российский рынок капитала, который в свою очередь является развивающимся, а также будет произведено перестроение глобальной межвременной модели ценообразования активов в локальную. Данная модель будет учитывать различные экономические особенности страны, влияющие на акции компаний нефинансового сектора. Поэтому, во-первых, будут найдены макропеременные, которые могут оказывать влияние на доходность акций российских компаний. Во-вторых, согласно Кэмпбеллу [Campbell, 2013], при помощи найденных в предыдущем пункте факторов риска и векторной авторегрессии будет произведена декомпозиция доходности рынка на составляющие (денежный поток, ставку дисконтирования), а также при помощи стохастического дисконтного фактора определена волатильность рынка. В-третьих, согласно Фаме и Френчу [Fama, French, 2004], каждая компания нефинансового сектора России будет включаться в один из 12 портфелей: таким образом, будет проведена процедура кластеризации акции российских компаний. В заключение будут рассчитаны риск-премии по каждому портфелю, где в качестве риск-факторов будут использоваться три коэффициента чувствительности каждого портфеля к изменениям в денежном потоке, ставке дисконтирования и волатильности.

\section{Методология}

В данной работе использована межвременная модель ценообразования активов, которая включает в себя не только рыночные риски, в том числе и валютный, но также волатильность российского рынка в целом. Интерес в использовании этой модели заключается в том, что ICAPМ имеет несколько преимуществ перед моделью, ориентированной на потребление. Вопервых, она описывает риски при их мгновенном появлении, чтобы инвестор, покупая любой актив, смог удовлетворить свое бюджетное ограничение. Именно так риски представлены в отдельных агентах экономики, и важно понимать поведение участников рынка, а не полагаться исключительно на макроэкономическую точку зрения. Во-вторых, ICAРМ генерирует эмпирические предсказания, которые зависят от коэффициента относительного неприятия риска. В-третьих, ICAРМ обеспечивает эмпирический анализ, основанный на прокси-переменных от совокупного рыночного портфеля, а не на точном измерении совокупного потребления. Хотя есть, конечно же, проблемы в точности измерения финансового благосостояния и временных рядов. В-четвертых, ICAРМ является достаточно гибкой моделью, что позволяет множеству стационарных переменных быть представленными в системе VAR, а также учитывает нелинейную зависимость основных макропеременных российского рынка от валютного курса.

Джон Кэмпбелл в 1993 году, используя разложение доходности на составляющие факторы будущих денежных потоков (дивиденды или потребление) и ставки дисконтирования (ожидаемая доходность), пришел к выводу, что исходные параметры в целом не описывают волатильность рынка [Campbell, 2013]. Но в соответствии с фундаментальной теоремой ценообразования финансовых активов отсутствие арбитражных возможностей эквивалентно существованию стохастического дисконтного фактора $\mathrm{m}$ - параметра, уравнивающего ожидаемый доход по активу в соответствии с рисковыми и временными предпочтениями инвестора. Поэтому в 2013 году Джон Кэмпбелл с соавторами выводят уравнение ценообразования ICAРМ с фактором волатильности рынка, используя стохастический дисконтный фактор и предпочтения Эпштейна-Зина [Campbell, 2013]. Объясняя данную модификацию модели тем, что первоначально, используя условие гомоскедастичности, когда коэффициент неприятия риска равен $1,-$ дисперсия суммы стохастического дисконтного фактора и доходности актива постоянна. Но данное предположение позже оказалось неверным ввиду того, что стохастический дисконтный фактор, изменяясь во времени, не зависит от коэффициента неприятия риска, а только от логлинеаризационного параметра $p$, который определяет долю инвестируемых средств, в зависимости от потребления и благосостояния инвестора. В результате после всех модификаций и выкладок модель приобретает следующий вид: 


$$
\begin{aligned}
& \mathrm{E}_{t} r_{i, t+1}-r_{f, t}+\frac{1}{2} \operatorname{Var}_{t} r_{i, t+1}=\gamma \operatorname{Cov}_{t}\left[r_{i, t+1}, r_{t+1}\right]+(\gamma-1) \operatorname{Cov}_{t}\left[r_{i, t+1}, N_{D R, t+1}\right]- \\
& \frac{1}{2} \operatorname{Cov}_{t}\left[r_{i, t+1}, N_{R I S K, t+1}\right]=\gamma \operatorname{Cov}_{t}\left[r_{i, t+1}, N_{C F, t+1}\right]+\operatorname{Cov}_{t}\left[r_{i, t+1},-N_{D R, t+1}\right]- \\
& \frac{1}{2} \operatorname{Cov}_{t}\left[r_{i, t+1}, N_{R I S K, t+1}\right]
\end{aligned}
$$

Авторы используют уже известное разбиение рыночной доходности на денежный поток и ставку дисконтирования [Campbell, Vuolteenaho, 2004]. А также добавляют третье слагаемое, отражающее подверженность доходности возникающим рискам по каждому активу. Текущая модифицированная модель ценообразования имеет премию за риск по фактору денежного потока в $\gamma$ раз больше, чем по фактору ставки дисконтирования. Поэтому Камбелл и Вуолтинахо в своей работе дают им соответствующие наименования «bad betta» и «good betta», т.к. последняя величина имеет более низкую премию за риск [Campbell, Vuolteenaho, 2004]. В итоге полученное уравнение объясняет ожидаемую доходность активов в зависимости от изменений в денежном потоке, ставки дисконтирования и будущих рисков репрезентативного инвестора.

Для реализации описанной выше модели будем следовать Джону Кэмпбеллу [Campbell, 2013] для определения шоков, влияющих на доходность рынка, используя модель векторной авторегрессии первого порядка (VAR):

$x_{(t+1)}=a+\Gamma x_{t}+\sigma_{t} u_{(\mathrm{t}+1)}$,

где $x_{(t+1)}-6$ х 1 вектор от основных переменных, $r_{t+1}$ - первый элемент данного вектора, $a$ и $\Gamma$ - постоянный вектор 6 x 1 и матрица от постоянных параметров размерности $\mathrm{N}$ x $\mathrm{N}$ соответственно.

Мы предполагаем, что первым элементом вектора $x_{t+1}$, состоящего из шести исходных стационарных переменных, будет доходность рынка. А остальные пять величин важны для прогнозирования будущей доходности рынка. Далее определим вектор , в котором на первом месте записана единица, а остальные элементы данного вектора - нули. Используя данный вектор и вектор стационарных переменных , можно определить реальную доходность рынка: $r_{m, t+1}: r_{m, t+1}=e_{1}^{\prime} x_{t+1}$, , и $x_{t+1}-\mathrm{E}_{t} x_{t+1}=e_{1}^{\prime} \sigma_{t} u_{t+1}$. Поэтому доходность рынка в любой момент времени может быть извлечена из VAR:

$\mathrm{E}_{t} r_{m, t+1+j}=e_{1}^{\prime} \Gamma^{j} x_{t}$

Учитывая эту структуру, фактор ставки дисконтирования и будущего риска запишем в следующем виде:

$$
\begin{aligned}
& N_{D R, t+1} / \sigma_{t}=\left(E_{t+1}-\mathrm{E}_{t}\right) \sum_{j=1}^{\infty} \rho^{j} r_{t+1+j}=e_{1}^{\prime} \sum_{j=1}^{\infty} \rho^{j} \Gamma^{j} u_{t+1}=e_{1}^{\prime} \rho \Gamma(I-\rho \Gamma)^{-1} u_{t+1} \\
& N_{r i s k, t+1}=\left(E_{t+1}-\mathrm{E}_{t}\right) \sum_{j=1}^{\infty} \rho^{j} \operatorname{Var}_{t+j}\left[r_{t+1+j}+m_{t+1+j}\right]=\omega \rho e_{2} \sum_{j=0}^{\infty} \rho^{j} \Gamma^{j} u_{t+1}= \\
& \omega \rho e_{2}^{\prime} \Gamma(I-\rho \Gamma)^{-1} u_{t+1}=\omega N_{V, t+1}
\end{aligned}
$$

Далее получим эмпирически проверяемую межвременную модель ценообразования:

$$
\begin{aligned}
& \mathrm{E}_{t} r_{i, t+1}-r_{f, t}+\frac{1}{2} \operatorname{Var}_{t} r_{i, t+1}=\gamma \operatorname{Cov}_{t}\left[r_{i, t+1}, N_{C F, t+1}\right]+\operatorname{Cov}_{t}\left[r_{i, t+1},-N_{D R, t+1}\right]- \\
& \frac{1}{2} \omega \operatorname{Cov}_{t}\left[r_{i, t+1}, N_{V, t+1}\right]
\end{aligned}
$$

где параметр $\omega$ - это нелинейная функция относительно коэффициента неприятия риска $\gamma$. Далее запишем $N_{C F}$ и $N_{V}$ через параметры VAR модели: 
$\frac{N_{C F, t+1}}{\sigma_{t}}=\left(e_{1}^{\prime}+e_{1}^{\prime} \rho \Gamma(I-\rho \Gamma)^{-1}\right) u_{t+1}$,

$\tilde{\mathbb{N}}_{\mathrm{V}, t+1} \mu / \sigma_{t}=e_{2}^{\prime} \rho \Gamma(I-\rho)^{-1}{ }_{t+1}$.

Преобразуем полученное уравнение ценообразования (3) в более известную форму, умножив и разделив все три ковариации по выборочной дисперсии на логарифм от ожидаемой реальной доходности рыночного портфеля. Поступая таким образом, мы можем выразить наше уравнение ценообразования с точки зрения бета, облегчая сравнения с предыдущими исследованиями. Эти изменения приводят к следующему уравнению ценообразования:

$$
\mathrm{E}\left[R_{i}-R_{f}\right]=\gamma \sigma_{M}^{2} \beta_{i, C F_{M}}+\sigma_{M}^{2} \beta_{i, D R_{M}}-\frac{1}{2} \omega \sigma_{M}^{2} \beta_{i, V_{M}} u_{t+1},
$$

где

$$
\begin{aligned}
& \beta_{i, C F_{M}}=\frac{\operatorname{Cov}\left(r_{i, t}, N_{C F, t}\right)}{\operatorname{Var}\left(r_{M, t}-E_{t} r_{M, t}\right)} \\
& \beta_{i, D R_{M}}=\frac{\operatorname{Cov}\left(r_{i, t}, N_{D R, t}\right)}{\operatorname{Var}\left(r_{M, t}-E_{t} r_{M, t}\right)} \\
& \beta_{i, V_{M}}=\frac{\operatorname{Cov}\left(r_{i, t}, N_{V, t}\right)}{\operatorname{Var}\left(r_{M, t}-E_{t} r_{M, t}\right)}
\end{aligned}
$$

Теперь мы переходим к расчету риск-премий, используя сквозную регрессии (cross sectional): $\overline{R_{i}^{e}}=g_{0}+g_{1} \widehat{\beta_{i, C F_{M}}}+g_{2} \widehat{\beta_{i, D R_{M}}}+g_{3} \widehat{\beta_{i, V_{M}}}+\varepsilon_{i}$, где $\overline{R_{i}^{e}}=\overline{R_{i}}-\overline{R_{r f}}-$ средняя выборочная избыточной доходности по каждому активу $i$.

Для эффективного анализа и проведения расчетов был составлен следующий алгоритм, который связывает все этапы данного исследования, перечисленные выше:

1. Следуя статье [Campbell, 1993a], вычислим VAR-модель первого порядка в уравнении (2), где $x_{t+1}-6 x 1$ вектор от основных переменных, в следующем порядке:

2. $x_{t+1}=($ Market, EVAR, ExRate, brent, CDS, Spread $)$

3. После проведения векторной авторегрессии первого порядка мы получаем коэффициенты данной модели, а именно константу а и матрицу постоянных коэффициентов $Г$.

4. Далее находим факторы денежного потока, ставки дисконтирования (определенные путем декомпозиции рыночной доходности), а также волатильности рынка: $N_{C F}, N_{D R}$, $N_{V}$

5. В качестве следующего шага считаем коэффициенты чувствительности рынка к изменению в денежном потоке, ставке дисконтирования и волатильности по каждой i-й компании: $\beta_{i, C F_{M}}, \beta_{i, D R_{M}}, \beta_{i, C F_{M}}$.

6. Далее мы строим сквозную регрессию (cross-sectional) для каждого портфеля по следующей формуле: $\overline{R_{i}^{e}}=g_{0}+g_{1} \widehat{\beta_{i, C F_{M}}}+g_{2} \widehat{\beta_{i, D R_{M}}}+g_{3} \widehat{\beta_{i, V_{M}}}+\varepsilon_{i}$.

Для расчетов используются ежемесячные данные с января 2005 года по декабрь 2013 года. При этом доходность каждого временного ряда посчитана как разница двух логарифмов в момент времени $t$ и $t$-1. Такая трансформация позволяет более наглядно представить связь между рассматриваемыми показателями, так как логарифмические ряды расположены в пределах единого диапазона.

Для проведения тестирования сначала необходимо выделить факторы, влияющие на доходность компаний. Предположим, что этими факторами будут служить: доходность рынка РТС, волатильность лаговых значений всех остальных переменных от дисперсии рынка, инфляция, 
обменный курс валют, цены на нефть Brent, кредитный дефолтный своп и спред временной доходности. Выбор данных переменных не случайный. Россия - крупный экспортер нефти, и ее экономика связана с мировыми ценами на нефть, так как доходы от продажи поступают в казну государства в долларах. А также российскими исследователями были получены существенные результаты в части оценки влияния нефтяных цен на российский фондовый рынок [Fedorova, Pankratov, 2009]. Авторы осуществили данную процедуру путем изменения рыночной стоимости нефтяных акций, составляющих значительную долю индекса РТС. Таким образом, в модели учтены следующие переменные: цена на нефть Brent, обменный курс валюты и индекс РТС. Далее в модель был включен показатель «кредитный дефолтный своп» (CDS), который, в частности, необходим инвестору для хеджирования рисков неплатежа по займам. И заключительная переменная - EVAR, которая отражает волатильность рынка от всех его лаговых значений остальных стационарных переменных.

Поэтому полная спецификация вектора $x_{t+1}$, входящего в VAR модель, включает шесть основных переменных, три из которых - такие же, как у Кэмпбелла, Гиглио и Полка [2011]. К этим переменным мы добавили следующие: обменный курс валюты - exchange rate, кредитный дефолтный своп (credit default swap) и цену на нефть марки Brent. Данные были взяты из Thomson data stream, помесячно с 1 января 2005 года по 31 декабря 2013 года. Теперь остановимся более подробно на каждой из переменных, тем самым опишем характеристику данных и способ вычисления доходностей по ним.

Первая переменная VAR модели (Market) - это разница между доходностью рынка РTC и инфляцией. Вторая переменная модели (Brent) - это доходность цен на Brent нефть. Третья переменная модели (CDS) - это кредитный дефолтный своп. Четвертая переменная модели (ExRate) - это финансовый обменный курс валюты. Следующей переменной в модели выступал «спред временной доходности», определялся данный показатель следующим образом: были найдены цены на долгосрочные и краткосрочные государственные облигации (где долгосрочные - это пятилетние, а краткосрочные - это трехмесячные) и вычислялась разность между доходностью долгосрочных облигаций и краткосрочных. И последняя переменная ожидаемое отклонение рынка, вычислялся этот показатель следующим образом: сначала была найдена переменная RVAR - «realized variance» дневной доходности в каждый момент времени $t$, затем была запущена регрессия от лаговых значений и остальных шесть макропеременных модели. В итоге определялось ожидаемое отклонение рынка: $\mathrm{EVAR}_{\mathrm{t}} \equiv \widehat{\mathrm{RVAR}_{\mathrm{t}+1}}$, тем самым был получен вектор макропеременных: $\mathrm{x}_{\mathrm{t}+1}=(\mathrm{RM}, \mathrm{EVAR}$, brent, CDS, ExRate, SpBond $)$.

\section{Эмпирическое тестирование модели}

Изначально была предпринята попытка протестировать данную модель на российском рынке акции без формирования портфелей. Получившиеся результаты не оправдали ожиданий, хотя риск-премия по ставке дисконтирования оказалась положительной; значение t-статистики по риск-премии денежного потока значимо на 1\%-ном уровне; коэффициент при бете ставки дисконтирования тоже значим на 5\%-ном уровне; но риск-премия волатильности оказалась незначимой. Еще пара отличий текущего тестирования от предыдущего заключались в следующем: инфляция была переменной, входившей в вектор VAR модели, а переменная VS, которую определили Кеннет Френч и Юджин Фама, являлась спредом между двумя портфелями, построенными на рынке акций США. Результаты проведения предыдущих эмпирических тестов показали, что для достоверной оценки премий за риск необходимо использовать показатель доходности портфелей. Поэтому было выдвинуто предложение: используя опыт построения портфелей Юджина Фамы и Кеннета Френча, провести кластеризацию российских компаний на 12 портфелей.

Поэтому было произведено разбиение 102 нефинансовых компаний России на 12 портфелей следующим образом: для начала отсортировали компании по BE/ME и ME так, чтобы 25\% компаний, имеющих самый высокий коэффициент BE/ME, и $30 \%$, имеющих наименьший показатель ME, попали в портфель Small Value (см. табл. 1). 
Классификация российских компаний нефинансового сектора на 12 портфелей

\begin{tabular}{|c|c|c|c|}
\hline & $<=30 \mathrm{ME}$ percentil & & $>=70 \mathrm{ME}$ percentile \\
\hline & Small & Medium & Big \\
\hline$>=75 \mathrm{BE} / \mathrm{ME}$ percentile & Growth & Growth & Growth \\
\hline & Neutral Growth & Neutral Growth & Neutral Growth \\
\hline & Neutral Value & Neutral Value & Neutral Value \\
\hline$<=25 \mathrm{BE} / \mathrm{ME}$ percentile & Value & Value & Value \\
\hline
\end{tabular}

Далее для проведения расчетов использовали статистический пакет STATA 12 и при помощи функций Excell нашли остатки векторной авторегрессии, факторы денежного потока, ставки дисконтирования и волатильности, посчитали ковариацию между ними и каждым портфелем и определили коэффициент чувствительности (бета) портфеля к изменениям в найденных факторах.

Таблица 2

Модель VAR (1) - GARCH (1,1)

Итоговая таблица по расчету влияния всех факторов ВАР модели от своих лаговых значений

\begin{tabular}{|c|c|c|c|c|c|c|}
\hline \multirow{2}{*}{\multicolumn{2}{|c|}{$\begin{array}{c}\text { Sample: } 2005 \mathrm{~m} 1-2013 \mathrm{~m} 12 \\
\text { Distribution: Gaussian }\end{array}$}} & \multirow[b]{4}{*}{ EVAR } & \multirow{2}{*}{\multicolumn{3}{|c|}{$\begin{array}{l}\text { Number of obs }=\quad 107 \\
\text { Wald chi2(36) }=4169,23\end{array}$}} & \\
\hline & & & & & & \\
\hline \multicolumn{2}{|c|}{ Log likelihood $=1776,01$} & & Prob $>$ & $=0$ & & \\
\hline & Market & & EX_RATE & BRENT & CDS & SP_BOND \\
\hline Market_L1 & $-0,003$ & $-0,003$ & $-0,012$ & 0,435 & $-0,006$ & 0,001 \\
\hline EVAR_L1 & $-4,278$ & 0,601 & 0,4016 & $-3,556$ & 0,242 & 0,039 \\
\hline EX_RATE_L1 & 0,1298 & $-0,006$ & $-0,041$ & 0,788 & $-0,002$ & $-0,001$ \\
\hline BRENT_L1 & 0,2814 & $-0,0001$ & $-0,081$ & $-0,110$ & $-0,012$ & 0,003 \\
\hline CDS_L1 & 2,1621 & $-0,040$ & 0,220 & 0,169 & 0,798 & $-0,002$ \\
\hline SP_BOND_L1 & $-1,277$ & 0,039 & $-0,025$ & 0,316 & 0,053 & 0,951 \\
\hline Constant & 0,029 & 0,004 & $-0,004$ & 0,037 & 0,0001 & 0,0007 \\
\hline
\end{tabular}

Следующий этап, согласно алгоритму, приведенному выше, - это построение векторной авторегрессии по лаговым значениям с гетероскедастичностью остатков модели, т.е. модель VAR (1) - GARCH $(1,1)$. Тестирование проводилось при помощи пакета STATA 12. Результат представлен ниже в таблице 2. А также произвели расчет месячной доходности по портфелю, которая равнялась средневзвешенной доходности каждой компании, входившей в данный портфель.

Из полученных результатов оценки коэффициентов VAR-модели получили необходимую матрицу Г, которая представлена таблицей 2, для вычисления факторов денежного потока, ставки дисконтирования и волатильности. Таблица 2 дает возможность сделать следующие выводы по переменным вектора и в целом по модели. В модели VAR $(1)$ - GARCH $(1,1)$ мерой качества регрессии служит не $R$-квадрат, а тест Вальда, т.к. используется метод максимального правдоподобия. Итак, огромное значение статистики Вальда в таблице 2 (4169), свидетельствует о том, что регрессия значима.

Таблица 3

Расчет риск-премий

\begin{tabular}{ccccc}
\hline & Коэффициенты & Стандартная ошибка & $\boldsymbol{t}$-статистика & $\boldsymbol{P}$-значение \\
constant & $-0,068$ & 0,019 & $-3,553$ & 0,007 \\
CF & 0,121 & 0,048 & 2,473 & 0,038
\end{tabular}


Илья Кучин $\mid$ Ilia Kuchin $27-38$ гов $62-75$

Затем провели регрессионный анализ методом наименьших квадратов в части поиска рискпремий по каждому фактору риска (денежного потока, ставки дисконтирования и волатильности), продемонстрированный в таблице 3. При помощи сквозной регрессии (cross-sectional) производится поиск риск-премий. Зависимой переменной выступает разница между средней доходностью портфеля и средней безрисковой процентной ставкой за весь исследуемый период, а независимыми переменными - коэффициенты чувствительности портфеля к изменениям в денежном потоке, ставки дисконтирования и волатильности рынка.

Анализируя таблицу 3, можно сделать следующий вывод: риск-премия по каждому фактору положительная. Рассмотрим значение $t$-статистики. Коэффициент при бете денежного потока значим на 5\%-ном уровне, т.к. Probability ( $t$-statistic) равно 0,035, а коэффициент при бете ставки дисконтирования незначим, т.к. p-value равно 0,09; что же касается коэффициента при бете волатильности, то данный коэффициент значим на 5\%-ном уровне.

\section{Заключение}

В настоящей работе представлена локальная межвременная модель ценообразования активов, в которой ожидаемая доходность активов определялась через взаимосвязь доходности рынка с факторами риска.

Тестирование модели проводилось на данных за 2005-2013 годы по 102 нефинансовым российским компаниям. В итоге подтверждается сильная спецификация межвременной модели ценообразования активов.

Определили шесть макропеременных, которые предсказывают ожидаемую доходность российского рынка. Для построения факторов риска произвели декомпозицию доходности российского рынка на денежный поток и ставку дисконтирования. При помощи стохастического фактора дисконтирования и дисперсии доходности рынка вычислили волатильность российского рынка. В качестве факторов риска выступали величины, демонстрирующие чувствительность доходности рынка к изменениям денежного потока, ставке дисконтирования и волатильности. Риск-премии по трем факторам риска получились значимыми. Хотя, сравнивая полученные риск-премии с работами предыдущих исследователей, можно сделать вывод, что премия волатильности имеет в несколько раз большее значение в данном исследовании. Это может быть связано с тем, что волатильность российского рынка существенно выше, чем в США. Также было замечено, что риск-премии по денежному потоку и ставке дисконтирования оказались больше, чем у Дж. Кэмпбелла. Таким образом, премии по локальной модели ожидаемо оказались выше, чем по глобальной.

\section{Список литературы}

1. Adler M., Dumas B. (1983) International portfolio choice and corporation finance: A synthesis. Journal of Finance, vol. 38, no. 3, pp. 925-984.

2. Agmon T. (1972) The relations among equity markets: A study of share price co-movements in the United States, United Kingdom, Germany and Japan. Journal of Finance, vol. 27, no. 4, 839-855.

3. Bekaert G, Harvey C.R., Ng A. (2005). Market integration and contagion. Journal of Business, vol. 78, no.1, 39-69.

4. Bekaert G, Harvey C.R. (1995) Time-varying world market integration. Journal of Finance, vol. 50, no. 2, pp. 403-444.

5. Blume M.E. (1971) On the assessment of risk. Journal of Finance, vol. 26, no. 2, pp. 1-10.

6. Bollerslev T. (1986) Generalized autoregressive conditional heteroscedasticity. Journal of Econometrics, vol. 31, no. 7, pp. 307-327. 
7. Brusa F., Ramadorai T., Verdelhan, A. (2014) The international CAPM Redux.

8. Carrieri F., Errunza V., Majerbi B. (2006), Does emerging market exchange risk affect global equity prices? Journal of Financial and Quantitative Analysis, vol. 41, no. 5, pp. 511-540.

9. Chen S.N. (1981) Beta nonstationarity, portfolio residual risk and diversification. Journal of Financial and Quantitative Analysis?, vol. 16, no. 4, pp. 95-111.

10. Chen S.W, Huang N.C. (2007) Estimates of the ICAPM with regime-switching betas: evidence form four pacific rim economies. Applied Financial Economics, vol. 17, no. 5, pp. 313-327.

11. Chi J., Li K., Young M. (2006) Financial integration in East Asian equity markets. Pacific Economic Review, vol. 11, no. 4, pp. 513-526.

12. De Santis G., Gerard B. (1997) International asset pricing and portfolio diversification with time-varying risk. Journal of Finance, vol. 52, no. 5, pp. 1881-1912.

13. De Santis G., Imrohoroglu S. (1997) Stock returns and volatility in emerging financial markets. Journal of International Money and Finance, vol. 16, no. 4, pp. 561-579.

14. Dumas B., Solnik B. (1995) The world price of foreign exchange risk. Journal of Finance, vol. 50 , no. 2 , pp. $445-479$.

15. Edwards S., Susmel R. (2001) Volatility dependence and contagion in emerging equity markets. Working Paper No. 8506. National Bureau of Economic Research: Cambridge.

16. Engle, R.F. (1982). Autoregressive conditional heteroscedasticity with estimates of the variance of UK inflation. Econometrica, vol. 50, no. 2, pp. 987-1008.

17. Fabozzi F.J, Francis J.C. (1978) Stability tests for alphas and betas over bull and bear market conditions. Journal of Finance, vol. 32, no. 8, pp. 1093-1099.

18. Ferson W.E., Harvey C.R. (1991) The variation of economic risk premiums. Journal of Political Economy, vol. 99, no. 3, pp. 385-415.

19. Ferson W.E., Korajczyk R.A. (1995) Do arbitrage pricing models explain the predictability of stock returns. Journal of Business, vol. 68, no. 8, pp. 309-349.

20. Forbes K.J, Rigobon R. (2002) No contagion, only interdependence: Measuring stock market comovements. Journal of Finance, vol. 57, no. 5, pp. 2223-2261.

21. Gerard B., Thanyalakpark K., Batten J.A. (2003) Are the Asian markets integrated? Evidence from the ICAPM. Journal of Economics and Business, vol. 55, no. 7, pp. 585-607.

22. Harvey C.R. (1991) The world price of covariance risk. Journal of Finance, vol. 46, no. 1, pp. 111-157.

23. Korajczyk R.A., Viallet C. (1989) An empirical investigation of international asset pricing. The Review of Financial Studies, vol. 2, no. 4, pp. 553-585.

24. Lessard D. (1974) World, national, and industry factors in equity returns. Journal of Finance, vol. 29 , no. 2 , pp. 379-391.

25. Levy R.A. (1971) On the short-term stationarity of beta coefficients. Financial Analysts Journal, vol. 27, no. 3, pp. 55-62.

26. Lintner J. (1965) The valuation of risk assets and the selection of riskly investments in stock portfolios and capital budgets, Review of Economics and Statistics vol. 47, no. 1, pp. 13-37.

27. Sharpe W. (1964) Capital asset prices: A theory of market equilibrium under conditions of risk. Journal of Finance, vol. 19, no. 3, pp. 425-442.

28. Solnik B. (1974) The international pricing of risk: An empirical investigation of the world capital market structure. Journal of Finance, vol. 29, no. 2, pp. 365-378.

29. Solnik B. (1977) Testing international asset pricing: Some pessimistic views. Journal of Finance, vol. 32, no. 2, pp. 503-512. 
30. Susmel R. (1999) Switching volatility in international equity markets. Available at: www. bauer.uh. edu/rsusmel/Academic/COVINEW.pdf (accessed 1 Julay 2009).

31. Vassalou M. (2000) Exchange Rate and Foreign Inflation Risk Premiums in Global Equity Return. Journal of International Money and Finance, vol. 19, no. 7, pp. 433-470.

32. Wu H. (2008) International asset pricing models: A forecasting evolutions. International Research Journal of Finance and Economics, vol. 15, no. 3, pp. 175-184.

\title{
CURRENCY RISK AND LOCAL INTERTEMPORAL ASSET PRICING MODEL. CASE OF RUSSIA
}

\author{
Ilia Kuchin, \\ Graduate student Higher School of Economics, \\ Russia,Moscow, Shabolovka str.,26.E-mail:ikuchin@hse.ru
}

\begin{abstract}
The research is concentrated on the issue of evaluating the risk factors impact on the Russian market asset pricing. John Campbell developed a global intertemporal asset pricing model that takes into account a number of risk factors in 2013. Emerging markets, including Russia, each possesses unique traits that make using global model risk factors in the local model complicated. Extra risks that are connected with developing countries market inefficiency, limited diversification possibilities, structural and institutional aspects command different key asset pricing influence factors and application of local models that estimate the demanded profitability per own capita. In particular, key interconnected Russian market indexes of the currency rate and oil pricing were chosen as risk factors for the Russian market. The model was tested using data on 102 non-financial Russian companies' during 2005-2013. The novelty in this study was as follows: first, the new model of assessment of foreign exchange risk factors influence the cost of equity capital; secondly, made allowance for the influence of the exchange rate risk on cash flow fluctuations in the discount rate and idiosyncratic volatility in intertemporal asset pricing models; and thirdly, the tests carried out intertemporal asset pricing models in the Russian market. The results confirmed a strong model specification that talks about the possibility of its use and need to consider macro factors in the formation of the investment portfolio for the long term.
\end{abstract}

Keywords: intertemporal CAPM, exchange risk, local model, MGARCH

JEL: G12

\section{References}

1. Adler M., Dumas B. (1983) International portfolio choice and corporation finance: A synthesis. Journal of Finance, vol. 38, no. 3, pp. 925-984.

2. Agmon T. (1972) The relations among equity markets: A study of share price co-movements in the United States, United Kingdom, Germany and Japan. Journal of Finance, vol. 27, no. 4, 839-855.

3. Bekaert G, Harvey C.R., Ng A. (2005). Market integration and contagion. Journal of Business, vol. 78, no.1, 39-69.

4. Bekaert G, Harvey C.R. (1995) Time-varying world market integration. Journal of Finance, vol. 50 , no. 2 , pp. 403-444.

5. Blume M.E. (1971) On the assessment of risk. Journal of Finance, vol. 26, no. 2, pp. 1-10.

6. Bollerslev T. (1986) Generalized autoregressive conditional heteroscedasticity. Journal of Econometrics, vol. 31, no. 7, pp. 307-327.

7. Brusa F., Ramadorai T., Verdelhan, A. (2014) The international CAPM Redux. 
8. Carrieri F., Errunza V., Majerbi B. (2006), Does emerging market exchange risk affect global equity prices? Journal of Financial and Quantitative Analysis, vol. 41, no. 5, pp. 511-540.

9. Chen S.N. (1981) Beta nonstationarity, portfolio residual risk and diversification. Journal of Financial and Quantitative Analysis?, vol. 16, no. 4, pp. 95-111.

10. Chen S.W, Huang N.C. (2007) Estimates of the ICAPM with regime-switching betas: evidence form four pacific rim economies. Applied Financial Economics, vol. 17, no. 5, pp. 313-327.

11. Chi J., Li K., Young M. (2006) Financial integration in East Asian equity markets. Pacific Economic Review, vol. 11, no. 4, pp. 513-526.

12. De Santis G., Gerard B. (1997) International asset pricing and portfolio diversification with time-varying risk. Journal of Finance, vol. 52, no. 5, pp. 1881-1912.

13. De Santis G., Imrohoroglu S. (1997) Stock returns and volatility in emerging financial markets. Journal of International Money and Finance, vol. 16, no. 4, pp. 561-579.

14. Dumas B., Solnik B. (1995) The world price of foreign exchange risk. Journal of Finance, vol. 50 , no. 2 , pp. $445-479$.

15. Edwards S., Susmel R. (2001) Volatility dependence and contagion in emerging equity markets. Working Paper No. 8506. National Bureau of Economic Research: Cambridge.

16. Engle, R.F. (1982). Autoregressive conditional heteroscedasticity with estimates of the variance of UK inflation. Econometrica, vol. 50, no. 2, pp. 987-1008.

17. Fabozzi F.J, Francis J.C. (1978) Stability tests for alphas and betas over bull and bear market conditions. Journal of Finance, vol. 32, no. 8, pp. 1093-1099.

18. Ferson W.E., Harvey C.R. (1991) The variation of economic risk premiums. Journal of Political Economy, vol. 99, no. 3, pp. 385-415.

19. Ferson W.E., Korajczyk R.A. (1995) Do arbitrage pricing models explain the predictability of stock returns. Journal of Business, vol. 68, no. 8, pp. 309-349.

20. Forbes K.J, Rigobon R. (2002) No contagion, only interdependence: Measuring stock market comovements. Journal of Finance, vol. 57, no. 5, pp. 2223-2261.

21. Gerard B., Thanyalakpark K., Batten J.A. (2003) Are the Asian markets integrated? Evidence from the ICAPM. Journal of Economics and Business, vol. 55, no. 7, pp. 585-607.

22. Harvey C.R. (1991) The world price of covariance risk. Journal of Finance, vol. 46, no. 1, pp. 111-157.

23. Korajczyk R.A., Viallet C. (1989) An empirical investigation of international asset pricing. The Review of Financial Studies, vol. 2, no. 4, pp. 553-585.

24. Lessard D. (1974) World, national, and industry factors in equity returns. Journal of Finance, vol. 29, no. 2, pp. 379-391.

25. Levy R.A. (1971) On the short-term stationarity of beta coefficients. Financial Analysts Journal, vol. 27, no. 3, pp. 55-62.

26. Lintner J. (1965) The valuation of risk assets and the selection of riskly investments in stock portfolios and capital budgets, Review of Economics and Statistics vol. 47, no. 1, pp. 13-37.

27. Sharpe W. (1964) Capital asset prices: A theory of market equilibrium under conditions of risk. Journal of Finance, vol. 19, no. 3, pp. 425-442.

28. Solnik B. (1974) The international pricing of risk: An empirical investigation of the world capital market structure. Journal of Finance, vol. 29, no. 2, pp. 365-378.

29. Solnik B. (1977) Testing international asset pricing: Some pessimistic views. Journal of Finance, vol. 32, no. 2, pp. 503-512.

30. Susmel R. (1999) Switching volatility in international equity markets. Available at: www. bauer.uh. edu/rsusmel/Academic/COVINEW.pdf (accessed 1 Julay 2009). 
31. Vassalou M. (2000) Exchange Rate and Foreign Inflation Risk Premiums in Global Equity Return. Journal of International Money and Finance, vol. 19, no. 7, pp. 433-470.

32. Wu H. (2008) International asset pricing models: A forecasting evolutions. International Research Journal of Finance and Economics, vol. 15, no. 3, pp. 175-184.

\title{
INTERRELATION BETWEEN PAYOUT AND FINANCING DECISIONS: EVIDENCE FROM EMERGING MARKETS
}

\author{
Artem Anilov, \\ Post-graduate, National Research University Higher School of Economics \\ Russia,Moscow, Shabolovka str., 26.E-mail: Tema_anilov@mail.ru \\ Nikita Pirogov, \\ Senior lecturer National Research University Higher School of Economics \\ Russia,Moscow, Shabolovka str.,26.E-mail:npirogov@hse.ru
}

\begin{abstract}
Financing and payout decisions generally affect company's economic performance: they have impact (both directly and indirectly) on the free cash flow and, thus, on company's and shareholders' value. Search for optimal capital structure and optimal payout policy strategy that are likely to maximize shareholders' utility resulted in the papers, dedicated to determinants of capital structure and payout policy. In such papers, one of the policies is usually treated as a determinant for another one. This bound does not let researchers to make some conclusions about existence or absence of interrelation between payout and financing choices. To capture this interrelation, simultaneous regression analysis should be performed. Researchers, though, cannot come up with unified conclusion about the existence and direction of such interrelation.

The absence of certain results as well as low level of research done on emerging markets make this topic rather relevant.

The results of recent research on the interrelation between payout and financing decisions are discussed in this paper. We also develop an econometric model that allows us to check the existence of interrelation in emerging markets and to compare the results to those obtained from developed markets.

The article contributes to the existed literature in the following directions: first, two debt variables are taken into account (total and long-term debt) as well as two payout policy variables (total payout and dividend payout). Second, macroeconomic variables are controlled. Third, the results obtained from the companies from emerging countries are compared to those obtained from developed markets.
\end{abstract}

Keywords: capital structure; payout policy; emerging markets; corporate finance; simultaneous regression JEL: G32, G35

\section{Introduction}

Capital structure and dividend policy are among of the most researched topics in corporate finance. In 1958 and 1961, Modigliani and Miller published two papers, dedicated to capital structure and dividend policy respectively. The main conclusions of these papers are those about irrelevance of financing and payout policies in terms of value creation under some assumptions (absence of corporate taxes, absence of transaction costs, and absence of information asymmetry) [Modigliani, Miller, 1958; Modigliani, Miller, 1961].

In the real world, these assumptions never hold and MM's theorems do not work. This means that financing and payout decisions actually may affect the company's value. By somehow adjusting capital structure and dividend policy, the management is able to reach the aim of value maximization. 